The Egyptian Journal of Hospital Medicine (July 2020) Vol. 80 (2), Page 798-802

\title{
Two Sofosbuvir Containing Regimens in Egyptian Hepatitis C Related Compensated Liver Cirrhosis: Impact on Clinical Outcome and Liver Stiffness \\ Nahed A Makhlouf ${ }^{1 *}$, Youssef M Swifee ${ }^{1}$, Mohamed M Darwish ${ }^{1}$, Nasr K. Khalil'2, Amal A. Mahmoud ${ }^{3}$, Ahmed Medhat ${ }^{1,2}$, Rasha O Refaie ${ }^{4}$, Zainab G Mahran ${ }^{1}$ \\ ${ }^{1}$ Department of Tropical Medicine and Gastroenterology, ${ }^{3}$ Department of Clinical Pathology, Faculty of Medicine, Assiut University, ${ }^{2}$ Assiut Viral Hepatitis Treatment Center, \\ ${ }^{4}$ Internal Medicine Department, Helwan University, Egypt. \\ Corresponding Author: Nahed Ahmed Makhlouf, E-mail: nahedmak@yahoo.com,Orcid: 0000-0003-2949-4369
}

\begin{abstract}
Background/Aim: The current study aimed to evaluate the impact of sustained virological response (SVR) after two sofosbuvir (SOF) containing regimens on the clinical outcomes, non invasive markers of hepatic fibrosis and liver stiffness. Patients and Methods: The study included 200 patients with compensated HCV cirrhosis. Group 1: received SOF and Ribavirin (RBV) for 6 months, Group 2: received SOF, Daclatsvir (DAC) and RBV for 3 months. Hepatitis C Virus Ribonucleic Acid (HCV RNA) by Polymerase Chain Reaction (PCR) was done at baseline, end of treatment (EOT), SVR12, SVR48. Model for end stage liver disease (MELD) score was calculated, the aspartate aminotransferase to platelet ratio index (APRI) and Fibrosis-4 (FIB-4) scores were done. FibroScan was done at baseline and SVR48. Results: SVR12 occured in $75 \%$ and $96 \%$ in group 1 and 2, respectively. There was improvement in both of APRI and FIB-4 scores at EOT in the two groups, and maintained to SVR48. A significant improvement in mean liver stiffness (LS) occurred at SVR48 compared to baseline in the study groups. Improvement in LS was observed more in group 2. There was a significant decrease in the mean value of MELD score in group 2 and non-significant decrease in group 1 at SVR48 in comparison to pre-treatment mean value.

Conclusion: SOF/DAC/RBV was effective treatment regimen in compensated liver cirrhosis. This regimen resulted in significant improvement in MELD score, and liver stiffness at SVR48.
\end{abstract}

Keywords: Sofosbuvir; Daclatsvir; Cirrhosis; Outcome; MELD; LS.

\section{INTRODUCTION}

Hepatitis $\mathrm{C}$ virus (HCV) infection is of growing international concern ${ }^{(1)}$. Individuals with HCV may experience chronic fatigue, depression and lower quality of life (2). HCV leads to cirrhosis, hepatocellular carcinoma (HCC), and liver transplantation. The HCVrelated disease load increases as the infected persons progress to advanced stage of liver disease ${ }^{(3)}$.

Pegylated interferon alfa (PEG-IFN- $\alpha$ ) and ribavirin (RBV) for 24 or 48 lead to sustained viral suppression (SVR) in 65\% among those with $\mathrm{HCV}$ genotype $4^{(4)}$. Another Egyptian study reported $60 \%$ SVR in Egyptian genotype 4 patients received interferon- $\alpha 2$ a plus ribavirin for 48 weeks ${ }^{(5)}$. Sofosbuvir (SOF) inhibit HCV NS5B viral polymerase and it was recommended by $\boldsymbol{F D A}$ (2013) for treatment of chronic hepatitis $C$ caused by genotypes $1-4^{(6)}$.

Understanding of $\mathrm{HCV}$ life cycle and viral enzymes ${ }^{(7)}$, have led to generation of new direct-acting antiviral agents (DAAs) ${ }^{(8)}$. Those included NS3/NS4A protease inhibitors, NS5B polymerase inhibitors and NS5A inhibitors with a broader spectrum ${ }^{(9)}$.

Daclatasvir (DAC) is indicated for use with sofosbuvir for therapy of treatment naïve or PEG-IFN$\alpha$ and RBV experienced cases with chronic HCV (G 1a or 3) infection, for treatment naïve G3 patients with compensated cirrhosis \pm addition of weight based RBV and for all HCV genotypes with decompensated liver cirrhosis or post liver transplant recurrent infection with initial low dose of $\mathrm{RBV}^{(10)}$.

Clinical trials have demonstrated the safety and efficacy of these SOF-based regimens, and their approval. However, to the best of our knowledge, few studies were done to evaluate effect of SVR on fibrosis regression especially in genotype 4 .

\section{AIM OF THE WORK}

To assess the effect of sustained virological response (SVR) after two SOF containing regimens on the clinical outcomes, non invasive markers of hepatic fibrosis and liver stiffness in Egyptian $\mathrm{HCV}$ compensated liver cirrhosis

\section{PATIENTS AND METHODS Populations:}

A prospective observational study was conducted on 200 patients with compensated HCV related liver cirrhosis (treatment-naive) (genotype 4). $\mathrm{HCV}$ treatment was given according to National 
Guidlines in two different periods according to available drugs in Egypt.

Group 1 (100 patients from January 2015 to March 2015) received SOF (400 $\mathrm{mg}$ once daily) and RBV (1000 mg/day if body weight $<75 \mathrm{~kg}$ or $1200 \mathrm{mg} /$ day if body weight $\geq 75 \mathrm{~kg}$ ) for 6 months. Group 2 (100 patients from February 2016 to March 2016) received SOF (400 mg once daily), DAC (60 mg once daily) and RBV (starting dose $600 \mathrm{mg} /$ day, increase as tolerated) for 3 months. Inclusion criteria, exclusion criteria, clinical and laboratory evaluations as previously described in details in Swifee et al. ${ }^{(11)}$.

In addition, FibroScan examination was done at baseline. Calculation of APRI score and FIB-4, and MELD score were done at baseline, EOT and at SVR12. Patients with SVR12 were subjected to regular follow-up for 9 months in the form of:

1-Full clinical evaluation, laboratory investigation (CBC and liver function tests) and abdominal ultrasound examination at 3 months and 9 months after achieving SVR12.

2- HCV RNA level at 9 months after achieving SVR12 (SVR48).

3- Serial calculation of APRI and FIB-4 scores and MELD score after achieving SVR12 till the end of follow up.

4- FibroScan examination at 9 months after achieving SVR12 (SVR48).

\section{Calculated scores:}

1- Indirect fibrosis markers: the APRI score ${ }^{(12)}$ and FIB4 score $^{(13)}$ were calculated. 2- Calculation of MELD score $^{(14)}$.

\section{FibroScan:}

Liver stiffness assessment were done for all participants with TE (Echosens, FibroScan 502, France). It was expressed in $\mathrm{kPa}$.

\section{Evaluation of Virological Response:}

Serum HCV-RNA was calculated using quantitative polymerase chain reaction assay (Cobas Amplicor, HCV Roche, Branchburg, NJ, USA, v 2.0, detection limit $15 \mathrm{IU} / \mathrm{mL}$ ). Virological Response in both regimens was determined according to Franciscus ${ }^{(15)}$. Sustained virological response 12 (SVR12): HCV RNA below the detectable limit $(<15 \mathrm{IU} / \mathrm{mL})$ at 12 weeks following the completion of therapy. Sustained virological response 48 (SVR48): HCV RNA below the detectable limit $(<15 \mathrm{IU} / \mathrm{mL})$ at 12 months after the completion of therapy.

\section{Ethical approval:}

The research was approved by the Ethical Committee of the Faculty of Medicine, Assiut University. All patients gave their written informed consent.

\section{Statistical analysis:}

Date entry and analysis were performed using SPSS (Statistical Package for the Social Sciences) Version 19.0, SPSS Inc., Chicago, Ill., USA. Data were displayed as number, percentage, mean and standard deviation. Chi-square test was used to compare between qualitative data. Quantitative variables were compared using Mann-Whitney test. Wilcoxon Signed Rank Test was done to compare quantitative variables before and after treatment in case of non-parametric data. P-value was considered statistically significant if $<0.05$. The percent of change was calculated as: $100 \times$ (value of variable at end of treatment - value of variable at baseline) / value of variable at baseline.

\section{RESULTS:}

Demographic data of the studied groups:

In all patient groups male and female were equally distributed. Mean age \pm SD (range) of group 1, and group 2 was, $55.64 \pm 7.73(33-69)$ and $57.40 \pm 7.06(41-70)$ years, respectively.

Table (1) shows baseline Child-Pugh, MELD, APRI and FIB-4 scores of the studied groups. A significant difference in the mean value of MELD score between both groups was found, where group 1 had the highest value. The mean values of APRI and FIB-4 scores in group 1 were higher in comparison to group 2 .

Table (1): Baseline Child-Pugh, MELD, APRI and FIB-4 scores of the studied groups

\begin{tabular}{|lccccc|}
\hline \multicolumn{1}{c}{ Variables } & Group 1 $(\mathbf{n}=\mathbf{1 0 0})$ & \multicolumn{2}{c|}{ Group 2(n= 100) } & P-value \\
\hline Child-Pugh Class**: & No. & $\mathbf{\%}$ & No. & \% & \\
\hline A5 & 36 & 36.0 & 56 & 56.0 & $0.005^{*}$ \\
\hline A6 & 58 & 58.0 & 44 & 44.0 & $0.048^{*}$ \\
\hline B7 & 6 & 6.0 & 0 & 0.0 & $0.029^{*}$ \\
\hline MELD score & $7.26 \pm 2.83$ & $6.45 \pm 2.62$ & $0.047^{*}$ \\
\hline APRI score & $1.71 \pm 0.68$ & $1.44 \pm 1.02$ & $<0.001^{*}$ \\
\hline FIB-4 score & $4.15 \pm 1.40$ & $3.48 \pm 1.92$ & $<0.001^{*}$ \\
\hline
\end{tabular}

APRI, AST to Platelet Ratio Index; FIB-4, fibrosis index based on four factors; MELD, model for end stage liver disease. * Significant difference

**Child-Pugh Class A includes A5 and A6; Child-Pugh Cass B includes B6, B7 and B8.

Group 1, patients received Sofosbuvir and Ribavirin for six months; Group 2, patients received Sofosbuvir, Daclatasvir and Ribavirin for three months. 
Table (2) shows the virological response in both regimens in the current study

Table (2): Virological Response in Different Regimens in the Present Study

\begin{tabular}{|c|c|c|c|c|c|}
\hline \multirow[t]{2}{*}{ Virological response } & \multicolumn{2}{|c|}{$\begin{array}{l}\text { Group } 1 \\
(n=100)\end{array}$} & \multicolumn{2}{|c|}{$\begin{array}{l}\text { Group } 2 \\
(n=100)\end{array}$} & \multirow[t]{2}{*}{ P-value } \\
\hline & No. & $\%$ & No. & $\%$ & \\
\hline ETR & 98 & 98.0 & 100 & 100.0 & 0.497 \\
\hline Relapse & 23 & 23.0 & 4 & 4.0 & $<0.001 *$ \\
\hline SVR12 & 75 & 75.0 & 96 & 96.0 & $<0.001^{*}$ \\
\hline SVR48 & 74I & 74.0 & 96 & 96.0 & $<0.001 *$ \\
\hline Virological failure \$ & 25 & 25.0 & 4 & 4.0 & $<0.001 *$ \\
\hline $\begin{array}{l}\text { ETR, end treatment response; SVR, sustained virological response. } \\
\text { Group 1, patients received Sofosbuvir and Ribavirin for six months; } \\
\text { and Ribavirin for three months. * Significant difference } \\
\text { II One patient of those who were achieved SVR } 12 \text { in Group } 1 \text { died b } \\
\text { shock as a result of haematemsis and hepatic encephalopathy. \$ incl }\end{array}$ & $\begin{array}{l}\text { Gro } \\
\text { befor } \\
\text { lude } t\end{array}$ & pati & $\mathrm{ec}$ & Sofos & ir, Daclata \\
\hline
\end{tabular}

Changes in the indirect fibrosis markers and MELD score:

a) Changes at the EOT compared to baseline in the study groups:

Improvement in APRI and FIB-4 scores at the EOT occurred in the study groups $(P$ value $<0.001$ for each). The marked decrease in the mean FIB-4 and APRI scores was noticed in group 1 compared to group 2 [the percent of improvement was $(-25.46$ versus 22.68) and (- 43.72 versus -39.88$)$ respectively].

There was worsening of MELD score mean value in both groups at the EOT compared to baseline (from $7.26 \pm 2.83$ to $8.47 \pm 2.25$ in group 1) and (from $6.45 \pm$
2.62 to $7.60 \pm 2.23$ in group 2$)(P$ value $<0.001$ for each).

b) Changes at the end of follow up period, in comparison to baseline in the study groups.

We found a significant decrease in the indirect fibrosis markers (APRI and FIB-4 mean score) at the EOT and this significant decrease continued during the follow-up period in both groups (Fig. 1) $(P<0.05$ for each). We noticed a significant decrease in the mean value of MELD score in Group 2 (from 6.45 to 5.68; p value $<0.001$ ) and non-significant decrease in group 1 in comparison to pre-treatment mean value (from 7.26 to 7.03 ; $p$ value $=0.292$ ) at the end of follow-up.

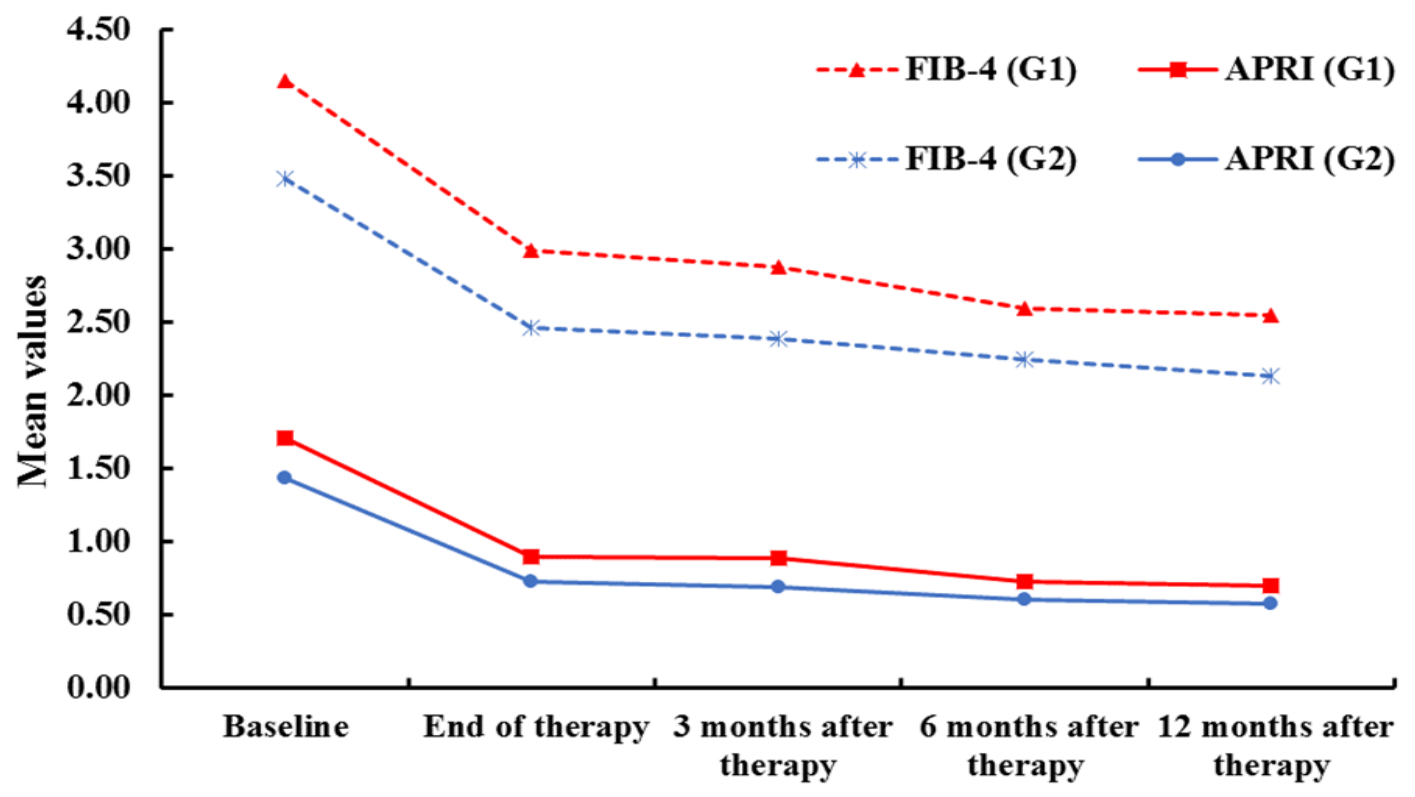

Figure (1): Changes in APRI and FIB-4 scores in both groups. 
Table (3) shows the changes in liver stiffness using FibroScan: In the present study, liver stiffness was determined in both groups at baseline by using FibroScan. No significant difference was found between both groups regarding the baseline mean liver stiffness. There was a significant improvement in mean liver stiffness value at SVR48 in comparison to baseline mean value in both groups (Table 3). The percentage improvement in liver stiffness was higher in group 2.

Table (3): Changes in Liver Stiffness score from Baseline to 12 months after the end of therapy (SVR48) using FibroScan

\begin{tabular}{|c|c|c|c|}
\hline $\begin{array}{c}\text { Liver Stiffness } \\
(\mathrm{kPa})\end{array}$ & $\frac{\text { Group 1 }}{\text { Mean } \pm \text { SD }}$ & $\begin{array}{c}\text { Group 2 } \\
\text { Mean } \pm \text { SD }\end{array}$ & P-value ${ }^{1}$ \\
\hline Baseline & $25.87 \pm 8.77$ & $22.85 \pm 6.58$ & 0.273 \\
\hline 12 months after therapy (SVR48) & $22.81 \pm 10.15$ & $18.03 \pm 5.28$ & 0.138 \\
\hline Mean difference & $-3.06 \pm 5.14$ & $-4.83 \pm 4.96$ & 0.283 \\
\hline Percent of difference & $-12.93 \pm 20.38$ & $-19.05 \pm 22.58$ & $0.045^{*}$ \\
\hline P-value ${ }^{2}$ & $0.014^{*}$ & $0.001 *$ & \\
\hline \multicolumn{4}{|c|}{$\begin{array}{l}\text { SVR, sustained virological response. } \\
\text { Group 1, patients received Sofosbuvir and Ribavirin for six months; Group 2, patients received Sofosbuvir, } \\
\text { Daclatasvir and Ribavirin for three months. } \\
\text { P-value }^{1} \text {, Compared between Group } 1 \text { and Group 2; P-value }{ }^{2} \text {, Compared baseline with } 12 \text { months after } \\
\text { therapy in each group (SVR48), * Significant difference }\end{array}$} \\
\hline
\end{tabular}

\section{DISCUSSION}

This prospective observational study was conducted on $200 \mathrm{HCV}$ related compensated LC (genotype 4) in two different periods of times. The SVR rate in group 1 patients who received $\mathrm{SOF} / \mathrm{RBV}$ was $75 \%$ (75/100). This was in concordance with the result of Abd-Elsalam et al. ${ }^{(16)}$ who found after treating 2265 treatment-naive and treatment-experienced Egyptian cirrhotic patients (Child A and B) with SOF/RBV for 6 months, the total SVR 12 rate was $71.2 \%$ while the SVR 12 rate among treatment-naive patients was $75.4 \%$. Also, Doss et al. ${ }^{(17)}$ reported that the SVR12 rate was highest in the group received treatment for 24 weeks (78\%) which was very similar to our results.

In the current study, $96 \%$ in group 2 who received (SOF/DAC/RBV) achieved SVR 12 which was similar to 94\% SVR12 reported by El-Khayat et al. (18) in treatment-naive patients with hepatitis C G4 related cirrhosis (CTP A and B) treated with SOF/DAC $\pm \mathrm{RBV}$.

Patients who achieved SVR12, had undetectable HCV RNA at SVR48, which was in agreement with Yoshida et al. ${ }^{(19)}$ who reported that, the SVR12 and SVR24 correlate closely in HCV genotypes 1-6 patients treated with SOF-based regimens, with or without IFN.

We found an increase in the mean value of MELD score at the EOT in comparison to pretreatment mean score in both groups. This can be explained by the increase in bilirubin level during treatment. At the end of follow-up, there was a significant decrease in the mean value of MELD score in group 2 and nonsignificant decrease in group 1 . This was in agreement with Poordad et al. ${ }^{(20)}$ who found decreases in the mean MELD score at 3 months following treatment of 60 patients with SOF/DAC/RBV for 12 weeks.

There was a significant reduction in the mean value of indirect fibrosis markers (APRI and FIB-4) at the EOT and this significant reduction continued during the follow-up period in the study groups. The marked reduction in the mean FIB-4 and APRI scores was noticed in group 1 . This was consistent with the results of Elsharkawy et al. ${ }^{(21)}$

There was a decrease in mean liver stiffness score at SVR48 (one year after the end of therapy) in comparison to baseline mean score in both groups. The above mentioned results were in agreement with Mahmoud et al. ${ }^{(22)}$ who found a significant decrease in the mean liver stiffness score at 6 months after EOT in comparison to pretreatment mean score by $2.01 \mathrm{kPa}$ in 200 Egyptian CHC patients (among them 124 patients were cirrhotic) received $\mathrm{SOF} / \mathrm{DAC} \pm \mathrm{RBV}$ for 12 weeks.

Also, in agreement with Elsharkawy et al. ${ }^{(21)}$ who found decreases in the liver stiffness mean level from baseline to SVR12.

Tag-Adeen (2017) demonstrated that HCV genotype-4 eradication results in reduction of $\operatorname{LSM}^{(23)}$. 
IN CONCLUSION: In cirrhotic patients who achieved SVR after two SOF containing regimen, we found an improvement in MELD score, APRI and FIB4 at the time of SVR12 and at SVR48 compared to baseline values. Also, liver stiffness measurements improved at SVR48 compared to baseline measurements.

Fund: No.

Conflict of interest: All authors declared that they had no conflict of interest.

Acknowledgments: The research team would like to thank all the staff members and specialist in Viral Hepatitis Treatment Center in Assiut.

\section{REFERENCES}

1. Stanaway JD, Flaxman AD, Naghavi M et al. (2016): The global burden of viral hepatitis from 1990 to 2013: findings from the Global Burden of Disease Study. Lancet; 388:1081-88.

2. Ibrahim EM and Madian A (2011): Impact of hepatitis $\mathrm{C}$ on health-related quality of life in Egypt. $J$ Am Sci., 7(11):430-439.

3. Chhatwal J, Wang X, Ayer T et al. (2016): Hepatitis C disease burden in the United States in the era of oral direct-acting antivirals. Hepatology; 64:1442-50.

4. European Association for the Study of the Liver (EASL) (2011): Clinical Practice Guidelines: management of chronic hepatitis $\mathrm{C}$ virus infection. $J$ Hepatol., 55:245-264.

5. El Makhzangy H, Esmat G, Said M et al. (2009): Response to pegylated interferon alfa-2a and ribavirin in chronic hepatitis C genotype 4. J Med Virol., 81:1576-83.

6. Lam AM, Murakami E, Espiritu C et al. (2010): PSI7851 , a pronucleotide of beta-D-2'-deoxy-2'-fluoro-2'-Cmethyluridine mono phosphate, is a potent and pangenotype inhibitor of hepatitis $\mathrm{C}$ virus replication. Antimicrob Agents Chemother., 54(8):3187-96.

7. Moradpour D, Penin F and Rice C (2007): Replication of hepatitis C virus. Nat Rev Microbiol., 5:453-463.

8. Buhler S and Bartenschlager R(2012): New targets for antiviral therapy of chronic hepatitis C. Liver Int., 32(1):19-16.

9. Sarrazin C, Hezode C, Zeuzem S and Pawlotsky $\mathbf{J}(2012)$ : Antiviral strategies in hepatitis $\mathrm{C}$ virus infection. J. Hepatol.,56(1):S88-S100.

10. AASLD/IDSA-USA HCV Guidance (2017): Recommendations for Testing, Managing, and Treating Hepatitis C. Available at: https://www.hcvguide lines.org.

11. Swifee YM, Makhlouf NA, Darwish MM et al. (2019). Impact of two sofosbuvir-containing regimens on the haematological and biochemical profiles of Egyptian patients with hepatitis C-related compensated cirrhosis. Journal of GHR; 8(1): 1-8.

12. Wai CT, Greenson JK, Fontana RJ et al. (2003): A simple noninvasive index can predict both significant fibrosis and cirrhosis in patients with chronic hepatitis C. Hepatology; 38:518-526.

13. Sterling RK, Lissen E, Clumeck $\mathrm{N}$ et al.(2006): Development of a simple noninvasive index to predict significant fibrosis patients with HIV/HCV co-infection. Hepatology, 43:1317-1325.

14. Kamath PS and Kim WR (2007): Advanced Liver Disease Study Group. The model for end-stage liver disease (MELD). Hepatology, 45:797-805.

15. Franciscus A (2015): www.hcvadvocate.org/hepatitis/factsheets pdf/ treatment_response_terms.pdf.

16. Abd-Elsalam S, Sharaf-Eldin M, Soliman $S$ et al. (2018).: Efficacy and safety of sofosbuvir plus ribavirin for treatment of cirrhotic patients with genotype 4 hepatitis $\mathrm{C}$ virus in real-life clinical practice. Arch Virol., 163:51-56.

17. Doss W, Shiha G, Hassany M et al. (2015): Sofosbuvir plus ribavirin for treating Egyptian patients with hepatitis C genotype 4. J Hepatol., 63:581-585.

18. El-Khayat H, Fouad Y, Mohamed HI et al. (2018): Sofosbuvir plus daclatasvir with or without ribavirin in 551 patients with hepatitis C-related cirrhosis, genotype 4. Aliment Pharmacol Ther., 47(5):674-679.

19. Yoshida EM, Sulkowski MS, Gane EJ et al. (2014): Concordance of sustained virological response 4,12 , and 24 weeks post-treatment with sofosbuvir-containing regimens for hepatitis C virus. Hepatol., 61(1):41-45.

20. Poordad F, Schiff ER, Vierling JM et al. (2016): Daclatasvir with sofosbuvir and ribavirin for hepatitis C virus infection with advanced cirrhosis or post-liver transplantation recurrence. Hepatology, 63:1493-505.

21. Elsharkawy A, Abdel Alem S, Fouad R et al. (2017): Changes in liver stiffness measurements and fibrosis scores following sofosbuvir based treatment regimens without interferon. J Gastroenterol Hepatol., 32:16241630.

22. Mahmoud HS, Osman HA, Abdelrazek GM et al. (2017): Evaluation of portal hypertension doppler parameters after hepatitis $\mathrm{C}$ virus eradication in patients with definite fibrosis. Int. J. Curr. Res. Med. Sci., 3(11):46-54.

23. Tag-Adeen M, Sabra AM, Akazawa Y et al. (2017): Impact of hepatitis $\mathrm{C}$ virus genotype-4 eradication following direct acting antivirals on liver stiffness measurement. Hepatic Medicine: Evidence and Research,9 45-53. 